\title{
The association of interpregnancy interval and mode of delivery with outcomes among a cohort of Guatemalan women with a history of prior cesarean
}

\section{Margo Harrison ( $\square$ MARGO.HARRISON@CUANSCHUTZ.EDU)}

University of Colorado Denver - Anschutz Medical Campus https://orcid.org/0000-0003-2940-2241

\section{Ana Garces}

Instituto de Nutricion de Centroamerica y Panama

\section{Lester Figueroa}

Instituto de Nutricion de Centroamerica y Panama

Jamie Westcott

University of Colorado Denver - Anschutz Medical Campus

Michael Hambidge

University of Colorado Denver - Anschutz Medical Campus

Nancy F Krebs

University of Colorado Denver - Anschutz Medical Campus

\section{Research}

Keywords: mode of delivery after cesarean, interpregnancy interval, Guatemala

Posted Date: August 7th, 2020

DOI: https://doi.org/10.21203/rs.3.rs-52175/v1

License: (c) (i) This work is licensed under a Creative Commons Attribution 4.0 International License.

Read Full License 


\section{Abstract}

Design: Our objectives were to analyze how interpregnancy interval (IPI) was associated with delivery mode and how outcomes varied by these characteristics.

Methods: This secondary analysis used data from a prospective study conducted in Chimaltenango, Guatemala from January 2017 through April 2020.

Results: Of 26,465 Guatemalan women, 3,170 (12.0\%) had a history of prior cesarean. $560(20.1 \%)$ women delivered by vaginal birth after cesarean with the remaining 2,233 $(79.9 \%)$ delivered by repeat cesarean delivery. Repeat cesarean reduced the risk of needing a dilation and curettage compared to vaginal birth after cesarean, but this association did not vary by IPI (AOR $0.01-0.03, p<0.001$ ). Repeat cesarean delivery, as compared to vaginal birth after cesarean, significantly reduced the likelihood a woman breastfeeding within one hour of birth (AOR $0.009-0.10, p<0.001$ ), but IPI was not associated with the outcome. Regarding stillbirth, repeat cesarean birth reduced the likelihood of stillbirth as compared to vaginal birth (AOR $0.2, p=0.001-0.002)$, but again IPI was not associated with the outcome.

Conclusion: Outcomes by mode of delivery among a Guatemalan cohort of women with a history of prior cesarean birth do not vary by IPI.

\section{Plain English Summary:}

During a qualitative study where providers at one public hospital in Guatemala were interviewed about their beliefs, attitudes, and general practices regarding mode of delivery among women with a history of prior cesarean birth, providers reported that women with a short interval pregnancy (less than 18 or 24 months depending on provider interviewed) were not candidates for trial of labor after cesarean in their facility due to risk of adverse outcomes. We wished to test the hypothesis that adverse pregnancy outcomes are no more likely with an interval of shorter than 18 or 24 months compared to a longer interval, regardless of whether a woman delivers by vaginal or cesarean birth. This analysis suggests that outcomes can be worse for infants with vaginal birth after cesarean as compared to elective repeat cesarean birth, but these outcomes do not vary by interpregnancy interval. Therefore, our hypothesis was correct.

\section{Introduction:}

During a qualitative study where providers at one public hospital in Guatemala were interviewed about their beliefs, attitudes, and general practices regarding mode of delivery among women with a history of prior cesarean birth, providers reported that women with a short interval pregnancy (less than 18 or 24 months depending on provider interviewed) were not candidates for trial of labor after cesarean in their facility (unpublished data, Harrison). Three prior studies have shown a risk of uterine rupture associated with pregnancy spacing among women with a history of prior cesarean delivery; the first reports an 
increased risk of rupture with an interdelivery interval less than 16 months; the second found an interval of less than 19 months was associated with a $2.25 \%$ risk of uterine rupture compared to a $1.05 \%$ risk with an interval longer than 19 months; and there was an increased odds of rupture at less than 18 months (AOR 3.0, Cl 1.3-7.2) compared to greater than 18 months (AOR 1.1, Cl 0.4-3.2) in the final analysis. ${ }^{1-3}$ Guidance from the American College of Obstetricians and Gynecologists (ACOG) discusses interpregnancy interval (IPI) in relation to likelihood of vaginal birth after cesarean success, but not related to pregnancy outcomes such as uterine rupture. The World Health Organization recommends 24 months between a livebirth and subsequent conception but does not give specific recommendations in the setting of a prior cesarean birth. ${ }^{4}$

Given the current common practice at this facility in Guatemala was not to offer trial of labor after cesarean if a woman delivered a baby in the past 18 or 24 months and lack of clear guidance on this topic, the aim of this analysis was to observe mode of delivery and associated maternal and perinatal/neonatal outcomes by IPI among women with a history of prior cesarean delivery in a large Guatemalan cohort. Our objectives were to analyze: 1) how IPI was associated with mode of delivery, and 2) how maternal and perinatal/neonatal outcomes varied by mode of delivery and IPI. We hypothesized (based on prior research) that repeat cesarean delivery would be associated with better neonatal outcomes than vaginal delivery after cesarean, but that these findings would not vary by IPI.

\section{Methods}

Study Design: This analysis was conducted using data from a prospective study conducted in communities in Chimaltenango, Guatemala from January 2017 through April 2020, through the Global Network for Women's and Children's Health Research, Maternal and Newborn Health Registry (MNHR). ${ }^{5}$

Setting: The Global Network's prospective registry, the MNHR, includes pregnancy related data and outcomes from rural or semi-urban geographical areas. The Guatemalan site includes 17 distinct clusters served by one referral hospital, 30 health centers, and 42 health posts. ${ }^{5}$ Each community generally represents the catchment area of a primary healthcare center, and about 300 to 500 annual deliveries. ${ }^{5}$ The objective of the MNHR is to enroll pregnant women as early as possible during the pregnancy and to obtain data on pregnancy outcomes for all deliveries of registered women, regardless of delivery location (i.e., home, health clinic, or hospital). ${ }^{5}$

Population: Only women with a history of prior cesarean delivery with information on date of last delivery and mode of delivery were included in this analysis. If date of last delivery or mode of delivery data was missing, women with a history of prior cesarean were not analyzed with the cohort.

Recruitment: The analyses presented here used MNHR data to determine mode of delivery among women with a history of prior cesarean by IPI as well as to analyze maternal and perinatal/neonatal outcomes of these deliveries, since 2017. The population studied included women screened for the MNHR who were eligible, consented, and delivered in the study period. ${ }^{5}$ Data were excluded from women who were 
enrolled but lost to follow-up prior to delivery, maternal deaths prior to labor and delivery, miscarriages, medically terminated pregnancies, and those with missing data for delivery mode or date of last delivery.

Primary Outcomes: The primary outcome of this analysis was mode of delivery among women with a history of prior cesarean, by IPI.

Secondary Outcomes: The secondary outcomes were maternal and perinatal/neonatal outcomes associated with mode of delivery among women with a history of prior cesarean, examined according to IPI.

Analysis Plan: We used descriptive statistics to produce counts and percentages on mode of delivery among women with a history of prior cesarean delivery. Then we observed independent variables associated with mode of delivery, and performed bivariate comparisons of sociodemographic and antenatal covariates, intrapartum characteristics, and maternal and perinatal/neonatal outcomes that we hypothesized might be associated with mode of delivery. P-values were obtained from bivariate comparisons as a function of each individual risk factor using Kruskal-Wallis, Fisher's Exact, or Chisquared tests depending on variable type.

All risk factors that occurred before delivery and might be associated with mode of delivery were including in a logistic regression associated with repeat cesarean delivery $(p<0.05$ from the individual risk factor bivariate comparisons). We performed this first considering IPI as a continuous variable and second as a dichotomous variable comparing an interval of $<12$ months vs $\geq 12$ months, a dichotomous variable comparing an interval of $<18$ months vs $\geq 18$ months, and a dichotomous variable comparing an interval of $<24$ months vs $\geq 24$ months.

We then used individual logistic regressions to observe the association of mode of delivery by IPI with maternal and perinatal/neonatal outcomes that were significantly different in bivariate comparisons by mode of delivery ( $p<0.05$ from the individual risk factor bivariate comparisons). Each of these outcomes was first considered using IPI as a continuous variable and second as a dichotomous variable comparing an interval of $<12$ months vs $\geq 12$ months, a dichotomous variable comparing an interval of $<18$ months vs $\geq 18$ months, and a dichotomous variable comparing an interval of $<24$ months vs $\geq 24$ months. No methods were used to adjust for any potential bias. All data analyses were performed with STATA software v.15.1. (STATA Corp, College Station, TX, USA).

Ethics: The appropriate institutional review boards/ethics research committees of the participating institutions approved the MNHR study (COMIRB \#08-0511, renewed 8/6/19). Individual informed consent for study participation was requested and obtained from each study participant. A Data Monitoring Committee, appointed by the National Institute of Child Health and Human Development reviewed the study semi-annually. ${ }^{5}$

\section{Results:}


Figure 1 is a flow diagram of the population of women included in this study. Between January 2017 and April 2020, 26,465 women delivered in the Guatemalan clusters of the MNHR. 3,170 women, 12.0\% of the MNHR population had a history of prior cesarean delivery. Of these women, 2,973 (88.1\%) had data available both on mode of delivery of the index pregnancy and the date of their prior delivery. About a fifth of these women $(560,20.1 \%)$ delivered by vaginal delivery after cesarean with the remaining 2,233 (79.9\%) delivering by repeat cesarean delivery.

Table 1 presents the sociodemographic and obstetric/labor characteristics of the population overall and by mode of delivery. The population was overall median age 27 with interquartile range (IQR) 23 to 31 years. Most women had some schooling (93.5\%), just over half were primiparous (52.7\%), and almost two-thirds women (73.1\%) were of normal or overweight body mass index (BMI). Women who delivered by repeat cesarean delivery (as compared to vaginal delivery after cesarean) were statistically more likely to be younger (median age 27 versus 28), to have had schooling (95.2\% versus $86.8 \%$ ), to be less parous (parity $3+11.6 \%$ versus $44.8 \%)$, and more likely to be overweight or obese $(71.0 \%$ versus $61.2 \%), p<$ 0.001 .

Figure 2 illustrates mode of delivery among women with a history of prior cesarean delivery as a function of IPI (time since a woman's last delivery). A univariate logistic regression of IPI (categorical variable of 6month intervals) on mode of delivery found that with each successive 6-month interval, repeat cesarean delivery became $20 \%$ more likely (UOR 1.2, p < 0.001).

Regarding obstetric and labor characteristics (Table 1) overall women had an IPI of 27 months (IQR 15 to 46 months), most had antenatal care (97.0\%) and singleton gestations (99.0\%), birthweight of their babies was median 2870 grams (IQR 2610 to 3120 grams) of mostly term infants (93.4\%). Only 1.6\% of the population was induced and a majority were delivered by obstetricians (83.0\%) in the hospital setting (81.3\%). When comparing women who delivered by repeat cesarean delivery compared to vaginal delivery after cesarean, they differed significantly on IPI (29 months versus almost 22 months), antenatal care (98.3\% versus $91.4 \%)$, women experiencing obstructed labor (4.5\% versus $2.3 \%$ ) and induction of labor (1.0\% versus $3.9 \%)$, hypertensive disease (7.1\% versus $1.1 \%)$, and referral in labor (19.2\% versus $10.0 \%), p$ $<0.05$. Women delivered by repeat cesarean delivery were more likely to be delivered by an obstetrician (96.9\% versus $27.3 \%$ ) and in the hospital (93.0\% versus $34.8 \%), p<0.001$.

Table 3 shows multivariable modeling of mode of delivery including all variables occurring prior to delivery significant in bivariate comparisons (age, education, parity, BMI, prenatal care attendance, IPI, obstructed labor, hypertensive disease, induction of labor, referred to the facility from another delivery setting, delivery attendant, and delivery in the hospital). The table shows the results of variables significant in the multivariable model as well as IPI, which was not significant (AOR 1.0, CI [1.0,1.0]). Increasing parity and induction of labor (as compared to not being induced) were associated with a reduced odds of repeat cesarean delivery (AOR 0.2, $p<0.05$ ] and AOR 0.1, p < 0.05). Increasing BMI category (AOR 1.4, $p<0.05$ ), experiencing hypertensive disease (AOR 3.5, $p<0.05$ ), and delivering in the hospital (AOR 3223.2, $p<0.05$ ) were associated with an increased risk of repeat cesarean. Delivery 
provider was dropped from the model as only physicians (compared to non-physicians) performed cesarean delivery.

Table 4 focuses on our objective of understanding how IPI is associated with mode of delivery in varying adjusted models. Compared to an interval of $<12$ months, if a women has $\geq 12$ months between pregnancies, she is no more likely to experience a repeat cesarean delivery (AOR 1.7, $p<0.05)$; the results were similar for women waiting $\geq 24$ months (AOR 1.3, $p<0.05$ ). However, for women waiting $\geq 18$ months compared to $<18$, they were found to have an increased risk of repeat cesarean delivery compared to vaginal delivery after cesarean (AOR 1.4, $p<0.05)$.

Table 5 shows the results of bivariate comparisons of maternal and perinatal/neonatal outcomes by mode of delivery. In the overall population most maternal outcomes were rare $(<2.0 \%)$, but $5.2 \%$ of women in the cohort were treated with magnesium sulfate for seizure prophylaxis and many women were treated with uterotonics (86.5\%). In bivariate comparisons, women varied by mode of delivery on uterotonic receipt ( $98.2 \%$ of repeat cesareans versus $40.0 \%$ of vaginal deliveries after cesarean) and dilation and curettage ( $0.1 \%$ of cesareans versus $3.2 \%$ of vaginal deliveries). With respect to neonatal outcomes, most babies were born alive (97.8\%), 21.1\% were breastfed within an hour of delivery, $4.1 \%$ of infants required neonatal antibiotics, and the remainder of adverse outcomes occurred rarely at less than a $2 \%$ prevalence. In bivariate comparisons, fetal status at delivery varied by mode of delivery (stillbirths in $1 \%$ of cesareans versus $3.9 \%$ of vaginal deliveries), as did breastfeeding within one hour of delivery $(6.1 \%$ of cesareans versus $82.9 \%$ of vaginal deliveries), $p<0.001$.

Table 6 presents the results of individual logistic regressions of the association of repeat cesarean delivery with the outcome of interest (those significant in bivariate comparisons) adjusted for IPI and covariates significant in bivariate comparisons. Maternal outcomes that varied by mode of delivery were uterotonic use, performance of dilation and curettage, and administration of magnesium sulfate. Each logistic regression was performed first with IPI as a continuous variable and then as a dichotomous variable (set at 12 months, 18 months, and 24 months). Results of each regression are presented by showing the independent association of both IPI and mode of delivery. Neither IPI nor mode of delivery was associated with uterotonic use or magnesium sulfate administration. Repeat cesarean delivery reduced the risk of needing a dilation and curettage, but this association did not vary by how IPI was defined (AOR $0.01-0.03, p<0.001)$.

Table 7 presents the results of individual logistic regressions of the association of repeat cesarean delivery with the outcome of interest (those significant in bivariate comparisons) adjusted for IPI and covariates significant in bivariate comparisons. Perinatal outcomes that varied by mode of delivery were breastfeeding within one hour and fetal status at delivery. Each logistic regression was performed first with IPI as a continuous variable and then as a dichotomous variable (set at 12 months, 18 months, and 24 months). Results of each regression are presented by showing the independent association of both IPI and mode of delivery. Regarding breastfeeding, repeat cesarean delivery as compared to vaginal delivery after cesarean significantly reduced the likelihood of a woman breastfeeding within one hour of delivery 
(AOR $0.009-0.10, p<0.001$ ), but IPI was not associated with the outcome across all definitions, although it nearly increased breastfeeding at intervals over 12 months compared to under a year (AOR 2.0, $p=0.06)$. Regarding stillbirth, repeat cesarean delivery reduced the likelihood of stillbirth as compared to vaginal delivery (AOR $0.2, p=0.001-0.002$ ), but IPI was not associated with the outcome across all definitions, although it marginally (but not statistically) reduced stillbirth at intervals of greater than compared to less than 12 months (AOR 0.3, $p=0.07$ ).

\section{Discussion:}

The main findings of this analysis were that there was an overall vaginal delivery after cesarean rate of $19.6 \%$ (that declined from $29.2 \%$ down to $16.3 \%$ over each successive six month interval between pregnancies, $p<0.001)$, and that repeat cesarean delivery reduced the odds of postpartum dilation and curettage, breastfeeding within one hour, and stillbirth as compared to vaginal delivery after cesarean. These associations did not change by IPI (time from last delivery to conception of incident pregnancy). Therefore, based on outcomes available in the MNHR and this analysis, women should not be precluded from attempting trial of labor after cesarean by IPI, alone.

The relationship between IPI and mode of delivery was significant in this analysis only when IPI was considered a dichotomous variable comparing less than 18-month intervals to greater than or equal to 18-month intervals (Table 4). Women with a longer interval in this analysis had $40 \%$ increased odds of repeat cesarean delivery, $p=0.03$. It is interesting that per our qualitative research (which represents only one facility), women only become eligible for trial of labor after 18 or 24 months, depending on the provider. A study from the Netherlands found that an IPI of less than 24 months is not associated with a decreased success of vaginal delivery after cesarean, but success rates decrease when the interval increases, which is what we found in our analysis as well. ${ }^{6}$ In a study of a population in California, USA, interdelivery intervals of less than 19 months were associated with a decreased rate of vaginal delivery after cesarean only in patients who underwent induction, but not for women in spontaneous labor. ${ }^{7}$ While we did not address induction of labor in our analysis, we also found that rates of successful vaginal delivery after cesarean were higher in women with shorter interval pregnancies (in an unadjusted comparison, Fig. 2) and in an adjusted comparison when the inflection point of the interval was 18 months (Table 4). We hypothesize that this finding is due to delivery location and spontaneity of labor and feel this is an area ripe for future research.

The finding that vaginal delivery after cesarean compared to repeat cesarean delivery is associated with an increased need for dilation and curettage is not a novel or surprising finding (Table 6). Retained placenta occurs in about $1-3 \%$ of vaginal deliveries compared to less than $1 \%$ of cesarean deliveries. ${ }^{8,9}$ What was important to note about this finding is the significance and odds of the complication did not vary by IPI. This finding suggests that ultrasound after vaginal delivery may be a clinical practice worth considering as part of a quality improvement program in this population. ${ }^{10,11}$ Our findings were similar regarding the odds of breastfeeding within one hour after delivery (Table 7) in that the outcome was not novel, and it did not vary by IPI. It is more common that women breastfeed earlier after a vaginal delivery 
than a cesarean, and again, if the population under study desires to address this issue, interventions targeted at this outcome exist for replication. ${ }^{12-15}$ It is notable that at the 12-month timepoint, an interval longer than a year was marginally (not statistically) associated with an increased odds of breastfeeding after delivery.

While no outcomes varied by mode of delivery with respect to IPI, the only borderline result concerned stillbirth. Women with an interval of greater than 12 months between delivery and subsequent conception had a marginal statistically significant decrease in stillbirth. This suggests that if providers want to counsel women on trial of labor with respect to IPI, they would be justified in citing a trend toward an increase in stillbirth at less than 12 months, but a recommendation against trial of labor for this reason would not be supported by the results of our analysis. While a concerning finding that vaginal delivery after cesarean increases the odds of stillbirth compared to repeat cesarean in this population, neonatal outcomes are already known to be better under these circumstances and thus, this is not a novel finding. ${ }^{16-19}$

This analysis is limited in lacking uterine rupture as a maternal outcome of relevance to the study question. However, the dataset includes relevant related covariates such as hysterectomy and blood transfusion as well as neonatal outcomes such as stillbirth. Other variables of interest that were unavailable but would contribute to a deeper understanding of this complex scenario include mode of delivery intention compared to actual mode of delivery. Strengths of the analysis are the large sample size, which contributes to external validity, the high quality of the data, and the breadth of antepartum, intrapartum, and postpartum variables that were included in the analysis.

\section{Conclusion:}

In conclusion, this analysis provides an evidence base that could change the paradigm of counseling in Guatemala with respect to the relationship between IPI and mode of delivery among women with a history of prior cesarean delivery. While not the only risk factor of interest in a highly complex decisionmaking process, this analysis provides evidence that IPI need not be a strict exclusion criterion for trial of labor after cesarean with respect to the most common maternal and perinatal/neonatal outcomes. Studying the association of this finding with trial of labor after cesarean rates would be an important area for future study.

\section{Abbreviations}

IPI

Interpregnancy interval

MNHR

Maternal and newborn health registry

\section{Declarations}




\section{Author Contributions:}

MSH conceived of the analytic plan with feedback and input from LF, AG, and NFK. MSH performed the analysis with feedback and oversight from LF, AG, JW, and NK. MSH wrote the manuscript with input from all authors.

\section{Declarations:}

The authors have no relationships to disclose that may be deemed to influence the objectivity of this paper and its review. The authors report no commercial associations, either directly or through immediate family, in areas such as expert testimony, consulting, honoraria, stock holdings, equity interest, ownership, patent-licensing situations or employment that might pose a conflict of interest to this analysis. Additionally, the authors have no conflicts such as personal relationships or academic competition to disclose. The findings presented in this paper represent the views of the named authors only, and not the views of their institutions or organizations. Our manuscript does not contain any individual level data but is an analysis of de-identified data of women who consented for their data to be collected.

\section{Data Availability:}

Data available on request due to privacy/ethical restrictions

\section{Funding:}

Funding for this project comes from the Eunice Kennedy Shriver National Institutes of Child Health and Human Development Women's Reproductive Health Research K12 award (5K12HD001271) and the Doris Duke Charitable Foundation.

\section{References}

1. Al-Zirqi I, Daltveit AK, Forsen L, Stray-Pedersen B, Vangen S. Risk factors for complete uterine rupture. Am J Obstet Gynecol. 2017;216(2):165.e161-165.e168.

2. Shipp TD, Zelop CM, Repke JT, Cohen A, Lieberman E. Interdelivery interval and risk of symptomatic uterine rupture. Obstet Gynecol. 2001;97(2):175-177.

3. Bujold E, Gauthier RJ. Risk of uterine rupture associated with an interdelivery interval between 18 and 24 months. Obstet Gynecol. 2010;115(5):1003-1006.

4. Organization WH. Report of a WHO technical consultation on birth spacing: Geneva, Switzerland 1315 June 2005. World Health Organization;2007.

5. Bose CL, Bauserman M, Goldenberg RL, et al. The Global Network Maternal Newborn Health Registry: a multi-national, community-based registry of pregnancy outcomes. Reprod Health. 2015;12(2):S1.

6. Rietveld AL, Teunissen PW, Kazemier BM, De Groot CJM. Effect of interpregnancy interval on the success rate of trial of labor after cesarean. J Perinatol. 2017;37(11):1192-1196. 
7. Huang WH, Nakashima DK, Rumney PJ, Keegan KA, Jr., Chan K. Interdelivery interval and the success of vaginal birth after cesarean delivery. Obstet Gynecol. 2002;99(1):41-44.

8. Moragianni VA, Aronis KN, Craparo FJ. Risk factors associated with retained placenta after cesarean delivery. Journal of perinatal medicine. 2011;39(6):737-740.

9. Perlman NC, Carusi DA. Retained placenta after vaginal delivery: risk factors and management. Int J Womens Health. 2019;11:527-534.

10. Namazov A, Elkabetz N, Ivshin E, et al. Routine ultrasonographic and hysteroscopic evaluations of women undergoing postpartum manual removal of placenta: a retrospective cohort study. Archives of Gynecology and Obstetrics. 2020:1-5.

11. Iqbal H, Khan MS, Muneeb A, Mirza WA. Diagnostic accuracy of ultrasound in detecting retained products of conception: A study from a tertiary care hospital in Karachi, Pakistan. Cureus. 2018;10(11).

12. Prior E, Santhakumaran S, Gale C, Philipps LH, Modi N, Hyde MJ. Breastfeeding after cesarean delivery: a systematic review and meta-analysis of world literature. The American journal of clinical nutrition. 2012;95(5):1113-1135.

13. Rowe-Murray HJ, Fisher JR. Baby friendly hospital practices: cesarean section is a persistent barrier to early initiation of breastfeeding. Birth. 2002;29(2):124-131.

14. Hazlewood K. The Benefits of Skin-to-Skin Care After Cesarean Birth. 2019.

15. Dudeja S, Sikka P, Jain K, Suri V, Kumar P. Improving first-hour breastfeeding initiation rate after cesarean deliveries: A quality improvement study. Indian pediatrics. 2018;55(9):761-764.

16. Kawakita T, Downs SG, Ghofranian A, Mokhtari N. 593: Neonatal outcomes of trial of labor after cesarean delivery compared with elective cesarean. American Journal of Obstetrics \& Gynecology. 2020;222(1):S380.

17. Obstetricians ACo, Gynecologists. ACOG Practice bulletin no. 115: Vaginal birth after previous cesarean delivery. Obstet Gynecol. 2010;116(2 Pt 1):450.

18. Grünebaum A, McCullough LB, Arabin B, Chervenak FA. Serious adverse neonatal outcomes such as 5-minute Apgar score of zero and seizures or severe neurologic dysfunction are increased in planned home births after cesarean delivery. PloS one. 2017;12(3).

19. Boyle A, Preslar JP, Hogue CJ, et al. Route of Delivery in Women With Stillbirth: Results From the Stillbirth Collaborative Research Network. Obstet Gynecol. 2017;129(4):693-698.

\section{Tables}

Table 1. Population characteristics of women with a history of prior cesarean birth overall and by mode of delivery, January 2017 - April 2020 


\section{Total Women with History}

of Cesarean

$N=2793$
Vaginal Birth After

Cesarean

$n=560,20.1 \%$
Repeat

Cesarean Birth

$\mathrm{n}=2233$,

$79.9 \%$

\section{Sociodemographics}

\begin{tabular}{|c|c|c|c|c|}
\hline Age in years [IQR] & $27[23,31]$ & $28[23,33]$ & $27[23,31]$ & $<0.001^{a}$ \\
\hline Schooling n, \% & & & & $<0.001^{c}$ \\
\hline Illiterate & $157,5.6 \%$ & $63,11.2 \%$ & $94,4.2 \%$ & \\
\hline Literate, no school & $24,0.9 \%$ & $11,2.0 \%$ & $13,0.6 \%$ & \\
\hline Schooling & $2612,93.5 \%$ & $486,86.8 \%$ & $2126,95.2 \%$ & \\
\hline Parity n, \% & & & & $<0.001^{c}$ \\
\hline 1 & $1472,52.7 \%$ & $161,28.8 \%$ & $1311,58.7 \%$ & \\
\hline 2 & $812,29.1 \%$ & $148,26.4 \%$ & $664,29.7 \%$ & \\
\hline $3+$ & $509,18.2 \%$ & $251,44.8 \%$ & $258,11.6 \%$ & \\
\hline BMI kg/m² & & & & $<0.001^{b}$ \\
\hline$<18.5$ & $10,0.4 \%$ & $0,0.0 \%$ & $10,0.4 \%$ & \\
\hline $18.5-24.9$ & $856,30.6 \%$ & $217,38.8 \%$ & $639,0.6 \%$ & \\
\hline $25-29.9$ & $1188,42.5 \%$ & $222,39.6 \%$ & $966,43.3 \%$ & \\
\hline 30 & $739,26.5 \%$ & $121,21.6 \%$ & $618,27.7 \%$ & \\
\hline
\end{tabular}

Obstetric Characteristics

IPI in months [IQR] $\quad 27.3[15.2,46.3]$

$21.7[12.9,39.2]$

29.1

$[16.2,48.1]$

$<0.001^{a}$

Antenatal Care n, $\% \quad$ 2708, 97.0\%

$512,91.4 \%$

$2196,98.3 \%$

$<0.001^{c}$

Multiple Gestation $\quad 27,1.0 \%$

n, \%

$4,0.7 \%$

$23,1.0 \%$

$0.63^{b}$

Female sex of baby $\quad 1386,49.6 \%$

n, \%

Missing

$3,0.1 \%$

$285,50.9 \%$

$1101,49.3 \%$

$0.10^{c}$

$2,0.4 \%$

$1,0.1 \%$

Birthweight in

$2870[2610,3120]$

2855 [2585,3105]

$5,0.9 \%$

2890

$7,0.3 \%$

Missing

$2609,93.4 \%$

Term Gestational

Age n, \%

$518,92.5 \%$

[2610,3120]

$0.21^{a}$

$2,0.1 \%$

$2091,93.6 \%$

$0.33^{c}$ 


\begin{tabular}{|c|c|c|c|c|}
\hline $\begin{array}{l}\text { Obstructed Labor } n \text {, } \\
\%\end{array}$ & $114,4.1 \%$ & $13,2.3 \%$ & $101,4.5 \%$ & $0.02^{c}$ \\
\hline $\begin{array}{l}\text { Antepartum } \\
\text { Hemorrhage n, \% }\end{array}$ & $6,0.2 \%$ & $1,0.2 \%$ & $5,0.2 \%$ & $1.0^{\mathrm{b}}$ \\
\hline $\begin{array}{l}\text { Hypertensive } \\
\text { Disease n, \% }\end{array}$ & $164,5.9 \%$ & $6,1.1 \%$ & $158,7.1 \%$ & $<0.001^{c}$ \\
\hline $\begin{array}{l}\text { Induction of Labor } \\
\mathrm{n}, \%\end{array}$ & $44,1.6 \%$ & $22,3.9 \%$ & $22,1.0 \%$ & $<0.001^{c}$ \\
\hline \multirow{2}{*}{$\begin{array}{l}\text { Referred in Labor } \mathrm{n} \text {, } \\
\%\end{array}$} & & & & $<0.001^{c}$ \\
\hline & $485,17.4 \%$ & $56,10.0 \%$ & $429,19.2 \%$ & \\
\hline Missing & $1,0.1 \%$ & $0,0.0 \%$ & $1,0.1 \%$ & \\
\hline \multicolumn{2}{|l|}{ Attendant n, \% } & & & $<0.001^{b}$ \\
\hline Family & $1,0.05 \%$ & $1,0.2 \%$ & $0,0.0 \%$ & \\
\hline Non-OB MD & $120,4.3 \%$ & $52,9.3 \%$ & $68,3.1 \%$ & \\
\hline \multirow{2}{*}{$\begin{array}{l}\text { Nurse/Nurse } \\
\text { Midwife }\end{array}$} & $4,0.1 \%$ & $4,0.7 \%$ & $0,0.0 \%$ & \\
\hline & $2318,83.0 \%$ & $153,27.3 \%$ & $2165,96.9 \%$ & \\
\hline OB & $3,0.1 \%$ & $3,0.5 \%$ & $0,0.0 \%$ & \\
\hline \multirow{2}{*}{$\begin{array}{l}\text { Self } \\
\text { TBA }\end{array}$} & $1,0.05 \%$ & $1,0.2 \%$ & $0,0.0 \%$ & \\
\hline & $346,12.4 \%$ & $346,61.8 \%$ & $0,0.0 \%$ & \\
\hline \multicolumn{2}{|l|}{$\begin{array}{l}\text { Delivery Location } \mathrm{n}, \\
\%\end{array}$} & & & $<0.001^{b}$ \\
\hline \multirow{2}{*}{$\begin{array}{l}\text { Clinic/Health } \\
\text { Center }\end{array}$} & $2,0.1 \%$ & $1,0.2 \%$ & $1,0.1 \%$ & \\
\hline & $351,12.6 \%$ & $351,62.7 \%$ & $0,0.0 \%$ & \\
\hline Home & $2272,81.3 \%$ & $195,34.8 \%$ & $2077,93.0 \%$ & \\
\hline Hospital & $168,6.0 \%$ & $13,2.3 \%$ & $155,6.9 \%$ & \\
\hline Other & & & & \\
\hline
\end{tabular}
a. kruskall-wallis
b. fishers exact
c: chi $^{2}$ 
Table 3. Multivariable logistic regression of the association of risk factors significant in bivariate comparisons of mode of delivery

\begin{tabular}{|lll|}
\hline & Odds Ratio & P-Value \\
\hline Odds of Repeat Cesarean Birth & & \\
\hline Increase in IPI of 1 month & 1.0 & 0.5 \\
\hline Increase in parity of 1 birth & 0.4 & $<0.001$ \\
\hline Increase in BMI of 1 category (ref: underweight) & 1.4 & $\mathbf{0 . 0 0 1}$ \\
\hline Induction of labor (ref: not induced) & 0.1 & $<0.001$ \\
\hline Hypertensive disease (ref: no disease) & 3.5 & $\mathbf{0 . 0 1}$ \\
\hline Delivery in hospital setting (ref: non-hospital setting) & 3223.2 & $<0.001$ \\
\hline
\end{tabular}

*only significant odds ratios and IPI (as continuous variable) shown; all variables included in model that were significant in bivariate comparisons of mode of delivery: age, education, parity, body mass index, prenatal care attendance, obstructed labor, hypertensive disease, induction of labor, referred to facility from another setting, birth attendant, delivery in hospital setting

Note: birth attendant was dropped for physician providers only providing cesarean birth with zero cell for non-physician providers

Table 4. Multivariable logistic regression of the association of varying IPIs (as a dichotomous variable) with mode of delivery (repeat cesarean birth); adjusted for parity, body mass index, induction of labor, hypertensive disease, and delivery setting

\begin{tabular}{|lll|}
\hline & Odds Ratio & P-Value \\
Odds of Repeat Cesarean Birth & & \\
\hline IPI 12 months (ref: $<12$ months) & 1.7 & 0.1 \\
\hline IPI 18 months (ref: $<18$ months) & 1.4 & 0.03 \\
\hline IPI 24 months (ref: $<24$ months) & 1.3 & 0.1 \\
\hline
\end{tabular}

Table 5. Maternal and perinatal outcomes of women with a history of prior cesarean birth overall and by mode of delivery, January 2017 - April 2020 
Total Women with

History of Cesarean

$N=2793$
Vaginal Birth After

Cesarean

$n=560,20.1 \%$
Repeat

Cesarean Birth

$\mathrm{n}=2233$,

$79.9 \%$

\section{Maternal Outcomes}

\begin{tabular}{|c|c|c|c|c|}
\hline Uterotonics n, \% & $2416,86.5 \%$ & $224,40.0 \%$ & $2192,98.2 \%$ & $<0.001^{b}$ \\
\hline $\begin{array}{l}\text { Blood Transfusion } n \text {, } \\
\%\end{array}$ & $29,1.0 \%$ & $4,07 \%$ & $25,1.1 \%$ & $0.49^{b}$ \\
\hline D\&C/Suction n, \% & $21,0.8 \%$ & $18,3.2 \%$ & $3,0.1 \%$ & $<0.001^{b}$ \\
\hline Magnesium n, \% & $146,5.2 \%$ & $6,1.1 \%$ & $140,6.3 \%$ & $0.58^{b}$ \\
\hline Hysterectomy n, \% & $14,0.5 \%$ & $1,0.2 \%$ & $13,0.6 \%$ & $0.33^{b}$ \\
\hline Severe Infection n, \% & $35,1.3 \%$ & $3,0.5 \%$ & $32,1.4 \%$ & $0.09^{b}$ \\
\hline \multirow{2}{*}{$\begin{array}{l}\text { Postpartum } \\
\text { Infection n, \% }\end{array}$} & $9,0.3 \%$ & $2,0.4 \%$ & $7,0.3 \%$ & \multirow[t]{2}{*}{$1.0^{\mathrm{b}}$} \\
\hline & $83,3.0 \%$ & $15,2.7 \%$ & $68,3.1 \%$ & \\
\hline Seizure n, \% & $3,0.1 \%$ & $0,0.0 \%$ & $3,0.1 \%$ & $1.0^{\mathrm{b}}$ \\
\hline Missing & $83,3.0 \%$ & $15,2.7 \%$ & $68,3.1 \%$ & \\
\hline \multirow{2}{*}{$\begin{array}{l}\text { Unplanned } \\
\text { Hospitalization n, \% } \\
\text { Missing }\end{array}$} & $23,0.8 \%$ & $6,1.1 \%$ & $17,0.8 \%$ & \multirow[t]{2}{*}{$0.43^{b}$} \\
\hline & $83,3.0 \%$ & $15,2.7 \%$ & $68,3.1 \%$ & \\
\hline \multirow{2}{*}{$\begin{array}{l}\text { Death by } 42 \text { Days } n \text {, } \\
\%\end{array}$} & $1,0.1 \%$ & $1,0.2 \%$ & $0,0.0 \%$ & \multirow[t]{2}{*}{$0.20^{\mathrm{b}}$} \\
\hline & $83,3.0 \%$ & $15,2.7 \%$ & $68,3.1 \%$ & \\
\hline
\end{tabular}

Neonatal Outcomes

Fetal Status n, \%

$<0.001^{b}$

Born Alive, Alive

$2732,97.8 \%$

$526,93.9 \%$

$2206,98.8 \%$

Born Alive, Neonatal

Demise

Stillbirth

$17,0.6 \%$

$12,2.1 \%$

$5,0.2 \%$

$44,1.6 \%$

$22,3.9 \%$

$22,1.0 \%$

$\begin{array}{lllll}\text { Bag \& Mask } & 31,1.1 \% & 9,1.6 \% & 22,1.0 \% & 0.21^{\mathrm{b}} \\ \text { Resuscitation n, \% } & 1,0.1 \% & 0,0.0 \% & 1,0.1 \% & \end{array}$

Missing

Breastfeed Within an $\quad 581,21.1 \%$

$446,82.9 \%$

$135,6.1 \%$

$<0.001^{c}$ 


\begin{tabular}{|lllll|}
\hline $\begin{array}{l}\text { Hour n, } \\
\text { neonatal Antibiotics }\end{array}$ & $114,4.1 \%$ & $16,2.9 \%$ & $98,4.4 \%$ & $0.10^{\mathrm{c}}$ \\
\hline CPAP n, \% & $7,0.3 \%$ & $1,0.2 \%$ & $6,0.3 \%$ & $0.30^{\mathrm{b}}$ \\
Missing & $2,0.1 \%$ & $1,0.2 \%$ & $1,0.1 \%$ & \\
\hline Oxygen n, \% & $125,4.5 \%$ & $18,3.2 \%$ & $107,4.8 \%$ & $0.11^{\mathrm{c}}$ \\
\hline $\begin{array}{l}\text { Ventilation } \mathrm{n}, \% \\
\text { Missing }\end{array}$ & $15,0.5 \%$ & $3,0.5 \%$ & $12,05 \%$ & $1.0^{\mathrm{b}}$ \\
\hline $\begin{array}{l}\text { Death by } 42 \text { Days } \mathrm{n}, \\
\%\end{array}$ & $1,0.1 \%$ & $0,0.0 \%$ & $1,0.1 \%$ & \\
\hline Missing & $39,1.4 \%$ & $10,1.8 \%$ & $29,1.3 \%$ & $0.31^{\mathrm{c}}$ \\
\hline
\end{tabular}
a. kruskall-wallis
b. fishers exact
c. chi $^{2}$

Note: all tests performed excluding missing data

Table 6. Individual logistic regressions of maternal uterotonic administration, dilation and curettage, and magnesium sulfate by varying IPIs and mode of delivery adjusted for parity, body mass index, induction of labor, hypertensive disease, and delivery setting 


\section{Odds of Needing Maternal Uterotonics}

IPI as continuous variable

Increase in IPI of 1 month

1.0

0.5

Repeat cesarean birth (ref: vaginal birth after cesarean)

2.1

0.1

IPI as dichotomous variable

IPI 12 months (ref: < 12 months)

1.7

0.4

Repeat cesarean birth (ref: vaginal birth after cesarean)

2.1

0.1

IPI as continuous variable

IPI 18 months (ref: < 18 months)

1.2

0.5

Repeat cesarean birth (ref: vaginal birth after cesarean)

2.1

0.1

IPI as continuous variable

IPI 24 months (ref: < 24 months)

1.0

0.9

Repeat cesarean birth (ref: vaginal birth after cesarean)

2.1

0.1

\section{Odds of Needing Maternal Dilation \& Curettage}

IPI as continuous variable

Increase in IPI of 1 month

1.0

0.4

Repeat cesarean birth (ref: vaginal birth after cesarean)

0.03

$<0.001$

IPI as dichotomous variable

IPI 12 months (ref: < 12 months)

0.4

0.4

Repeat cesarean birth (ref: vaginal birth after cesarean)

0.02

$<0.001$

IPI as continuous variable

IPI 18 months (ref: < 18 months)

1.4

0.5

Repeat cesarean birth (ref: vaginal birth after cesarean)

0.03

$<0.001$

IPI as continuous variable

IPI 24 months (ref: < 24 months)

1.6

0.4

Repeat cesarean birth (ref: vaginal birth after cesarean)

0.01

$<0.001$ 
IPI as continuous variable

$\begin{array}{lll}\text { Increase in IPI of } 1 \text { month } & 1.0 & 0.2\end{array}$

$\begin{array}{lll}\text { Repeat cesarean birth (ref: vaginal birth after cesarean) } & 0.3 & 0.4\end{array}$

IPI as dichotomous variable

IPI 12 months (ref: $<12$ months) $\quad 0.3 \quad 0.6$

$\begin{array}{lll}\text { Repeat cesarean birth (ref: vaginal birth after cesarean) } & 0.6 & 0.7\end{array}$

IPI as continuous variable

IPI 18 months (ref: $<18$ months) $\quad 2.2 \quad 0.2$

$\begin{array}{lll}\text { Repeat cesarean birth (ref: vaginal birth after cesarean) } & 0.3 & 0.4\end{array}$

IPI as continuous variable

IPI 24 months (ref: $<24$ months) $\quad 2.0 \quad 0.2$

$\begin{array}{lll}\text { Repeat cesarean birth (ref: vaginal birth after cesarean) } & 0.3 & 0.5\end{array}$

Table 7. Individual logistic regressions of breastfeeding and stillbirth by varying IPIs and mode of delivery adjusted for parity, body mass index, induction of labor, hypertensive disease, and delivery setting 


\section{Odds of Breastfeeding Within 1 Hour of Birth}

IPI as continuous variable

Increase in IPI of 1 month

1.0

0.5

Repeat cesarean birth (ref: vaginal birth after cesarean)

0.009

$<0.001$

IPI as dichotomous variable

IPI 12 months (ref: < 12 months)

2.0

0.06

Repeat cesarean birth (ref: vaginal birth after cesarean)

0.009

$<0.001$

IPI as continuous variable

IPI 18 months (ref: < 18 months)

1.1

0.5

Repeat cesarean birth (ref: vaginal birth after cesarean)

0.01

$<0.001$

IPI as continuous variable

IPI 24 months (ref: < 24 months)

1.2

0.2

Repeat cesarean birth (ref: vaginal birth after cesarean)

0.01

$<0.001$

\section{Odds of Stillbirth}

IPI as continuous variable

Increase in IPI of 1 month $\quad 1.0 \quad 0.6$

$\begin{array}{lll}\text { Repeat cesarean birth (ref: vaginal birth after cesarean) } & 0.2 & \mathbf{0 . 0 0 1}\end{array}$

IPI as dichotomous variable

IPI 12 months (ref: < 12 months)

0.3

0.07

Repeat cesarean birth (ref: vaginal birth after cesarean)

0.2

0.002

IPI as continuous variable

IPI 18 months (ref: < 18 months)

0.8

0.5

Repeat cesarean birth (ref: vaginal birth after cesarean)

0.2

0.001

IPI as continuous variable

IPI 24 months (ref: < 24 months)

0.9

0.7

Repeat cesarean birth (ref: vaginal birth after cesarean)

0.2

0.001 


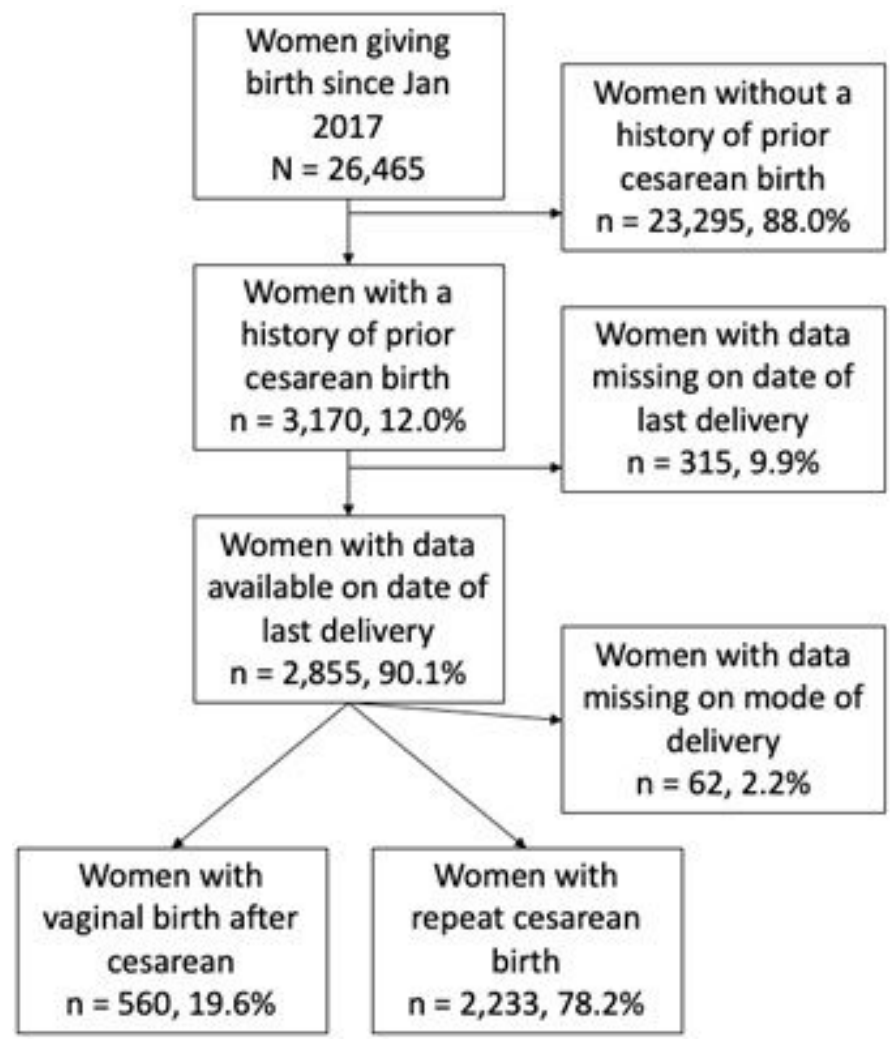

\section{Figure 1}

Population of women with a history of prior cesarean birth at the Guatemalan site of the Global Network for Women's and Children's Health Research, Maternal and Newborn Health Registry

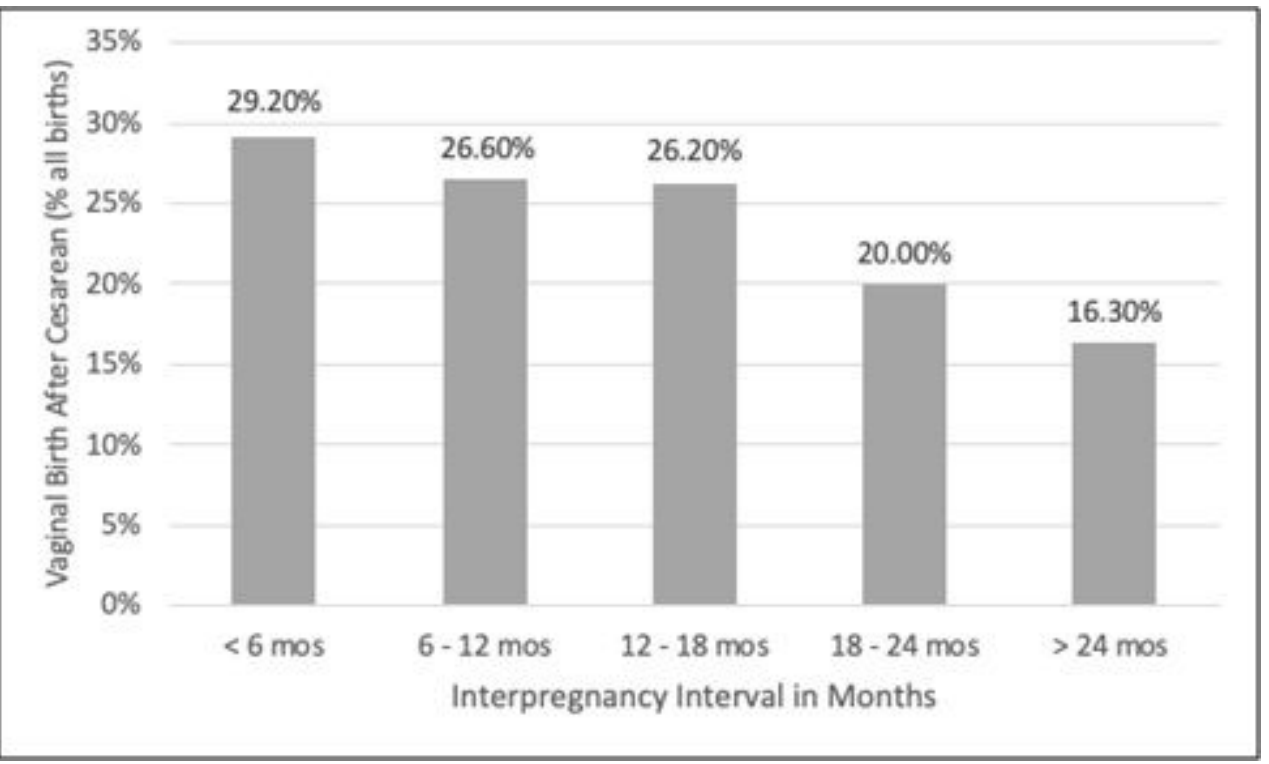

\section{Figure 2}

Proportion of vaginal birth after cesarean by 6-month IPIs with trend test Univariate logistic regression of time interval as categorical independent variable on mode of delivery: $20 \%$ increase in odds of repeat cesarean birth per six-month interval (UOR 1.2, CI [1.2,1.3], $\mathrm{p}<0.001)$ 